Pacific Journal of Mathematics

ON THE COMPACTNESS OF INTEGRAL CLASSES 


\title{
ON THE COMPACTNESS OF INTEGRAL CLASSES
}

\author{
WILLIAM P. ZIEMER
}

1. Introduction. In a previous paper, [8], integral currents were used to develop a concept for non-oriented domains of integration in Euclidean $n$-space. This concept has been designed to be useful in the calculus of variations and this, therefore, demands that the domains of integration satisfy certain "smoothness" and "compactness" conditions. It was shown in [8] that these non-oriented domains, which are called integral classes, do possess the desired smoothness property and it was also shown that the integral classes possess the following compactness property: every $N$-bounded sequence of $k$-dimensional integral classes has a subsequence which converges to some flat class. In the case that $k=0,1, n-1$, or $n$, it was shown that the limiting flat class is, in fact, a rectifiable class, and therefore, a desirable compactness property is obtained.

The main purpose of this paper is to extend this compactness property to integral classes of arbitrary dimension under the assumption that certain "irregular" sets have zero measure (3.1). This is accomplished with the help of a theorem concerned with the behavior of the density of a measure associated with a minimizing sequence (2.8), and by relying heavily on the tangential properties of rectifiable sets. In the case of the Plateau Problem, two theorems concerning densities are proved $(2.3,2.4)$ which are analogous to results obtained in [6] and [3; 9.13].

Most of this work depends upon the paper [8], and therefore, the terminology and notation of [8] is readopted here without change. It will be assumed throughout that $1<k<n-1$.

2. Densities. In this section, the Plateau Problem is formulated in terms of integral classes and two theorems are proved which are analogous to results obtained in [3; 9.13] and [6]. Theorem 2.8 asserts that a portion of the irregular set, $A_{3}$, which appears in (3) below, has zero measure. A similar result, which states that $D_{k}^{*}\left(\mu, R^{n}, x\right)<\infty$ $\mu$-almost everywhere and therefore that $\mu\left(A_{3}\right)=0$, is still lacking.

2.1. Definition. If $\mu$ is a measure over $R^{n}, A \subset R^{n}, \alpha(k)$ the volume of the unit $k$-ball, and $x \in R^{n}$, then

$$
D_{k}(\mu, A, x)=\lim _{r \rightarrow 0} \alpha(k)^{-1} r^{-k} \mu(A \cap\{y:|y-x|<r\})
$$

is the $k$-dimensional $\mu$ density of $A$ at $x$; the upper and lower densities

Received February 14, 1963. 


$$
D_{k}^{*}(\mu, A, x) \text { and } D_{* k}(\mu, A, x)
$$

are defined as the corresponding lim sup and lim inf.

2.2. REMARK. Recall that if $\left\{\tau_{i}\right\}$ is a sequence of integral classes with the property that $\sup \left\{M\left(\tau_{i}\right): i=1,2, \cdots\right\}<\infty$, then the sequence of total variation measures, $\left\{\left\|\tau_{i}\right\|\right\}$, possess a subsequence that converges weakly to some non-negative Radon measure $\mu,[8 ;(3.2)$, (3.3)], [2; Chapter III].

2.3. THEOREM. Suppose $\sigma \in I_{k-1}\left(R^{n}, 2\right)$ is a cycle and let

$$
\Omega(\sigma)=\inf \left\{M(\tau): \tau \in I_{k}\left(R^{n}, 2\right), \partial \tau=\sigma\right\} .
$$

Let $\left\{\tau_{i}\right\}$ be a sequence of integral classes such that

$$
\partial \tau_{i}=\sigma, \quad \lim _{i \rightarrow \infty} M\left(\tau_{i}\right)=\Omega(\sigma),
$$

and $\left\{\left\|\tau_{i}\right\|\right\}$ converges weakly to a non-negative Radon measure $\mu$. Then, for all $x \notin s p t \sigma$,

$$
D_{k}^{*}\left(\mu, R^{n}, x\right) \leqq \Omega(\sigma)\left(\alpha(k) r_{0}^{k}\right)^{-1}
$$

where $r_{0}=$ distance $(x$, spt $\sigma)$.

Proof. Let $\varepsilon_{i}$ be a sequence of real numbers tending to zero where

$$
\Omega(\sigma) \leqq M\left(\tau_{i}\right)<\Omega(\sigma)+\varepsilon_{i} .
$$

Let $B_{0}$ be the set of all $0<r<r_{0}$ with the property

$$
\begin{gathered}
\left\|\tau_{i}\right\|[S(x, r)] \rightarrow \mu[S(x, r)], \\
\operatorname{spt}\left[\partial\left(\tau_{i} \cap S(x, r)\right] \leqq\{y: \text { distance }(y, x)=r\},\right. \\
\tau_{i} \cap S(x, r) \text { is integral for } i=1,2,3, \cdots,
\end{gathered}
$$

and notice that $L_{1}\left[\left(0, r_{0}\right)-B_{0}\right]=0$. For $r \in B_{0}$ and $i=1,2,3, \cdots$,

$$
\tau_{i}=\tau_{i} \cap S(x, r)+\tau_{i} \cap\left[R^{n}-S(x, r)\right] .
$$

Letting

$$
\zeta_{i}=x \partial\left[\tau_{i} \cap S(x, r)\right]+\tau_{i} \cap\left[R^{n}-S(x, r)\right],
$$

[8; 3.14] implies

$$
\begin{gathered}
\partial \zeta_{i}=\partial \tau_{i}=\sigma, \\
M\left[x \partial\left(\tau_{i} \cap S(x, r)\right)\right] \leqq r / k M\left[\partial\left(\tau_{i} \cap S(x, r)\right)\right] .
\end{gathered}
$$

Therefore,

$$
M\left(\tau_{i}\right)<M\left(\zeta_{1}\right)+\varepsilon_{i},
$$


which implies

$$
\begin{aligned}
M\left[\tau_{i} \cap S(x, r)\right] & \leqq M\left[x \partial\left(\tau_{i} \cap S(x, r)\right)\right]+\varepsilon_{i} \\
& \leqq r / k M\left[\partial\left(\tau_{i} \cap S(x, r)\right)\right]+\varepsilon_{i},
\end{aligned}
$$

for $r \in B_{0}$ and $i=1,2,3, \cdots$. For $0<r<r_{0}$ and $i=1,2.3, \cdots$. let

$$
\begin{aligned}
& \varphi_{i}(r)=M\left[\tau_{i} \cap S(x, r)\right]=\left\|\tau_{i}\right\|[S(x, r)], \\
& \psi_{i}(r)=M\left[\partial\left(\tau_{i} \cap S(x, r)\right)\right],
\end{aligned}
$$

and note that $F_{i}(r)=\int_{0}^{r} \psi_{i}(t) d t \leqq \varphi_{i}(r)$, by [8; 4.1]. Again by [8; 4.1], $F_{i}(r+h)-F_{i}(r) \leqq \varphi_{i}(r+h)-\varphi_{i}(r)$ and therefore $F_{i}^{\prime}(r) \leqq \varphi_{i}^{\prime}(r)$ for $L_{1}$-almost all $0<r<r_{0}$. This implies

$$
\psi_{i}(r) \leqq \varphi_{i}^{\prime}(r)
$$

which, along with (1), implies

$$
\varphi_{i}(r) \leqq r k^{-1} \varphi_{i}^{\prime}(r)+\varepsilon_{i}
$$

for $i=1,2,3, \cdots$, and for $r \in B_{1} \subset B_{0}$ where $L_{1}\left(B_{0}-B_{1}\right)=0$.

After passing to a subsequence, we may assume by Helly's theorem that $\varphi(r)=\lim _{1 \rightarrow \infty} \varphi_{i}(r)$ exists whenever $0<r<r_{0}$, and therefore by $[3 ; 9.7]$, we have

$$
\lim _{i \rightarrow \infty} \inf \varphi_{i}^{\prime}(r) \leqq \varphi^{\prime}(r)
$$

for $r \in B_{2} \subset B_{1}$ where $L_{1}\left(B_{1}-B_{2}\right)=0$. Since

$$
\varphi_{i}(r) \leqq r k^{-1} \varphi_{i}^{\prime}(r)+\varepsilon_{i} \text { for } r \in B_{2},
$$

it follows that

$$
\varphi(r)=\lim _{i \rightarrow \infty} \varphi_{i}(r) \leqq \lim _{i \rightarrow \infty} \inf r k^{-1} \varphi_{i}^{\prime}(r) \leqq r k^{-1} \varphi^{\prime}(r)
$$

for $r \in B_{2}$. Therefore, for $L_{1}$-almost all $0<r<r_{0}$,

$$
\varphi^{\prime}(r) / \varphi(r) \geqq k / r \text {. }
$$

Letting $\theta(r)=\mu[S(x, r)]$, we have that $\theta(r)=\varphi(r)$ for $L_{1}$-almost all $0<r<r_{0}$ and thus, from (2), it follows that

$$
\theta^{\prime}(r) / \theta(r) \leqq k / r \text { for } L_{1} \text {-almost all } 0<r<r_{0} \text {. }
$$

Since $\log \circ \theta$ is non-decreasing, one finds by integrating this inequality that $\theta(r) r^{-k}$ is non-decreasing on $\left\{r: 0<\mathrm{r}<r_{0}\right\}$ and therefore, establishes the theorem.

2.4. THEOREM. With the same hypotheses and notations as in 
2.3, for $\mu$-almost $x \in R^{n}-s p t \sigma$,

$$
D_{* k}\left(\mu, R^{n}, x\right) \geqq\left(k \cdot \alpha(k)^{1 / k} \cdot 2^{k-1} \cdot c_{2}\right)^{-k}
$$

where $c_{2}$ is as in $[8 ; 4.6]$ with $k$ replaced by $k-1$.

Proof. Choose $x \nexists s p t \sigma$ so that $\varphi(r) \neq 0$ provided $r \neq 0$. For each $r \in B_{2}$, and for $i=1,2,3, \cdots$, from [8; 4.7] one obtains $\sigma_{i} \in I_{k}$ ( $\{y$ : distance $(x, y) \leqq r\}, 2)$ such that

$$
\begin{gathered}
\partial \sigma_{i}=\partial\left[\tau_{i} \cap S(x, r)\right], \\
{\left[M\left(\sigma_{i}\right)\right]^{k-1 / k} \leqq 2^{k-1} c_{2} \psi_{i}(r) .}
\end{gathered}
$$

Hence, $\varphi_{i}(r)<M\left(\sigma_{i}\right)+\varepsilon_{i}$ which implies

$$
\left[\varphi_{i}(r)\right]^{k-1 / k}<\left[M\left(\sigma_{i}\right)+\varepsilon_{i}\right]^{k-1 / k}<\left[M\left(\sigma_{i}\right)\right]^{k-1 / k}+\varepsilon_{i}^{*},
$$

where $\varepsilon_{i}^{*} \rightarrow 0$ for appropriate subsequences. From the fact that $\psi_{i}(r) \leqq \varphi_{i}^{\prime}(r)$ and from (1) and (2), we have

$$
\left[\varphi_{i}(r)\right]^{k-1 / k}<2^{k-1} c_{2} \varphi_{i}^{\prime}(r)+\varepsilon_{i}^{*}
$$

and therefore, from $[3 ;(9.7)]$

$$
[\varphi(r)]^{k-1 / k}=\lim _{i \rightarrow \infty}\left[\varphi_{i}(r)\right]^{k-1 / k} \leqq 2^{k-1} c_{2} \lim _{i \rightarrow \infty} \inf \varphi_{i}^{\prime}(r) \leqq 2^{k-1} c_{2} \varphi^{\prime}(r) .
$$

That is, for $L_{1}$-almost all $0<r<r_{0}$,

$$
\begin{aligned}
& {[\varphi(r)]^{k-1 / k} \leqq 2^{k-1} c_{2} \varphi^{\prime}(r),} \\
& {\left[\varphi^{1 / k}\right]^{\prime}(r) \geqq\left(k 2^{k-1} c_{2}\right)^{-1} .}
\end{aligned}
$$

Now, integration of this inequality implies

$$
\varphi(r) / r^{k} \geqq\left(k 2^{k-1} c_{2}\right)^{-k}
$$

and therefore establishes the theorem since $\varphi(r)=\mu[S(x, r)]=\theta(r)$ for $L_{1}$ almost all $0<r<r_{0}$, and since $\theta$ is left-continuous.

2.5. Lemma. If $\mu$ is a non-negative Radon measure over $R^{n}$, then for $\mu$-almost all $x \in R^{n}$,

$$
\lim _{r \rightarrow 0} \sup \frac{\mu[S(x, r / 2)]}{\mu[S(x, r)]}>0 \text {. }
$$

Proof. For $\mu$-almost all $x \in R^{n}$, we have

$$
\lim _{r \rightarrow 0} \mu[S(x, r)] \cdot r^{-m}=\infty
$$

where $m>n$. For all such $x$ the lemma must hold for, if not, there 
would exist an $r_{0}>0$ such that for $r \leqq r_{0}$,

$$
\frac{\mu[S(x, r / 2)]}{\mu[S(x, r)]}<2^{-m} \text {. }
$$

This would imply

$$
\frac{\mu\left[S\left(x, r 2^{-n}\right)\right]}{\left(r 2^{-n}\right)^{m}}=\frac{\mu\left[S\left(x, r 2^{-n}\right)\right]}{r^{m} 2^{-m n}} \leqq \frac{2^{-m n} \mu[S(x, r)]}{2^{-m n} r^{m}}=\frac{\mu[S(x, r)]}{r^{m}} ;
$$

hence, it would follow that

$$
\lim _{r \rightarrow 0} \inf \mu[S(x, r)] \cdot r^{-m}<\infty,
$$

a contradiction.

2.6. A Covering Theorem. From [1] and [5], we have the following theorem:

If $E \subset R^{n}, F$ is a family of closed spherical balls in $R^{n}$ such that each point of $E$ is the center of arbitrarily small members of $F$, and $\mu$ is a non-negative Radon measure over $R^{n}$, then $F$ has a disjointed subfamily covering $\mu$-almost all of $E$.

2.7. Definition. If $\tau \in W_{k}\left(R^{n}, 2\right)$, then let

$$
L(\tau)=\inf \left\{\lim _{i \rightarrow \infty} \inf M\left(\tau_{i}\right): \tau_{i} \in I_{k}\left(R^{n}, 2\right), \partial \tau_{i}=0, W\left(\tau_{i}-\tau\right) \longrightarrow 0\right\} .
$$

2.8. THeOREM. Suppose $\tau_{i} \in I_{k}\left(R^{n}, 2\right)$ are cycles for $i=1,2,3, \cdots$, $\left\{\left\|\tau_{i}\right\|\right\}$ converges weakly to a non-negative Radon measure $\mu$ over $R^{n}$, $\lim _{i \rightarrow \infty} W\left(\tau_{i}-\tau\right)=0$ and $\lim _{i \rightarrow \infty} M\left(\tau_{i}\right)=L(\tau)$ where $\tau \in W_{k}\left(R^{n}\right)$. Then, for $\mu$-almost all $x \in R^{n}$,

$$
D_{k}^{*}\left(\mu, R^{n}, x\right)>0 \text {. }
$$

Proof. The proof is by contradiction: by 2.5 we may assume the existence of a set $E$ and a real number $a>0$ such that for $x \in E$,

$$
\begin{gathered}
\mu(E)>0, \\
D_{k}^{*}\left(\mu, R^{n}, x\right)=0, \\
\lim _{r \rightarrow 0} \sup \frac{\mu[S(x, r / 2)]}{\mu[S(x, r)]}>a .
\end{gathered}
$$

Therefore, for a given $\varepsilon>0$ and for $x \in E$, there exists a set $B_{x} \subset(0,1)$ such that $B_{x}$ contains at least a denumerable number of elements and such that, if $r \in B_{x}$, 


$$
\begin{aligned}
& \mu[S(x, r)]<\varepsilon \alpha(k) r^{k}, \\
& \mu[S(x, r / 2)]>a \mu[S(x, r)], \\
& \mu[\{y: \text { distance }(x, y)=r\}]=0, \\
& \mu[\{y: \text { distance }(x, y)=r / 2\}]=0, \\
& \tau_{i} \cap S(x, r) \text { is integral for } i=1,2,3, \cdots, \\
& \inf \left\{r: r \in B_{x}\right\}=0 .
\end{aligned}
$$

Hence, by 2.6, there exist points $x_{1}, x_{2}, \cdots, x_{m}$ and numbers $r_{1}, r_{2}, \cdots$, $r_{m}$ such that $S\left(x_{i}, r_{i}\right) \cap S\left(x_{j}, r_{j}\right)=0$ for $i \neq j, r_{i} \in B_{x_{i}}$ and

$$
\mu\left[E-\bigcup_{j=1}^{m} S\left(x_{j}, r_{j}\right)\right]<\varepsilon .
$$

From (1), we have the existence of an integer $i_{0}>0$ such that for $i>i_{0}$ and $1 \leqq j \leqq m$,

$$
\begin{aligned}
& \left\|\tau_{i}\right\|\left[S\left(x_{j}, r_{j} / 2\right)\right]>a\left\|\tau_{i}\right\|\left[S\left(x_{j}, r_{j}\right)\right] \\
& \left\|\tau_{i}\right\|\left[S\left(x_{j}, r_{j}\right)\right]<\varepsilon \alpha(k) r_{j}^{k} .
\end{aligned}
$$

For $i \geqq i_{0}$ and $1 \leqq j<m,[8,4.1]$ provides a ball $S_{i, j} \subset S\left(x_{j}, r_{j}\right)$ with radius between $r_{j} / 2$ and $r_{j}$ and center at $x_{j}$ such that

$$
\begin{gathered}
\tau_{i} \cap S_{i, j} \text { is integral, } \\
M\left[\partial\left(\tau_{i} \cap S_{i, j}\right)\right] \leqq 2 r_{j}^{-1}\left\|\tau_{i}\right\|\left[S\left(x_{j}, r_{j}\right)\right] .
\end{gathered}
$$

Now by use of $[8 ; 4.6]$ one can find a constant $c$ and integral classes $\rho_{i, j}, \sigma_{i, j}$ such that,

$$
\begin{aligned}
& \tau_{i} \cap S_{i, j}-\rho_{i, j}=\partial \sigma_{i, j} \\
& M\left(\rho_{i, j}\right) \leqq c M\left[\partial\left(\tau_{i} \cap S_{i, j}\right)\right]^{k / k-1} \\
& M\left(\sigma_{i, j}\right) \leqq c\left[M\left(\tau_{i} \cap S_{i, j}\right)+M\left(\rho_{i, j}\right)\right]^{k+1 / k}
\end{aligned}
$$

For $i \geqq i_{0}$ and $1 \leqq j \leqq m,(2)$ and (3) imply

$$
\begin{aligned}
M\left[\partial\left(\tau_{i} \cap S_{i, j}\right)\right]^{k / k-1} & \leqq\left(2 r_{j}^{-1}\right)^{k / k-1} \cdot\left\|\tau_{i}\right\|\left[S\left(x_{j}, r_{j}\right)\right]^{k / k-1} \\
& \leqq\left(2 r_{j}^{-1}\right)^{k / k-1} \cdot\left\|\tau_{i}\right\|\left[S\left(x_{i}, r_{j}\right)\right] \cdot\left\|\tau_{i}\right\|\left[S\left(x_{j}, r_{j}\right)\right]^{1 / k-1} \\
& \leqq \varepsilon^{1 / k-1} \cdot 2^{k / k-1} \cdot \alpha(k)^{1 / k-1} \cdot\left\|\tau_{i}\right\|\left[S\left(x_{j}, r_{j}\right)\right]
\end{aligned}
$$

hence, from (4),

$$
M\left(\rho_{i, j}\right) \leqq \varepsilon^{1 / k-1} \cdot 2^{k / k-1} \cdot \alpha(k)^{1 / k-1} \cdot c \cdot\left\|\tau_{i}\right\|\left[S\left(x_{j} \cdot r_{j}\right)\right] .
$$

Similarly, 
(6) $M\left(\sigma_{i, j}\right) \leqq c\left[M\left(\tau_{i} \cap S_{i, j}\right)+M\left(\rho_{i, j}\right)\right]^{h+1 / k}$

$\leqq c\left\{\left\|\tau_{i}\right\|\left[S\left(x_{j}, r_{j}\right)\right]+\varepsilon^{1 / k-1} \cdot 2^{k / k-1} \cdot \alpha(k)^{1 / k-1} \cdot c \cdot\left\|\tau_{i}\right\|\left[S\left(x_{j}, r_{j}\right)\right]\right\}^{k+1 / k}$

$\leqq c\left\{\left(1+\varepsilon^{1 / k-1} \cdot 2^{k / k-1} \cdot \alpha(k)^{1 / k-1} \cdot c\right)\|\tau\|\left[S\left(x_{j}, r_{j}\right)\right]^{k / k+1} \cdot\left\|\tau_{i}\right\|\left[S\left(x_{j}, r_{j}\right)^{1 / k+1}\right\}^{k+1 / k}\right.$

$\leqq c\left\|\tau_{i}\right\|\left[S\left(x_{j}, r_{j}\right)\right] \cdot\left(1+\varepsilon^{1 / k-1} \cdot 2^{k / k-1} \cdot \alpha(k)^{1 / k-1} \cdot c\right)^{k+1 / k}\left\|\tau_{i}\right\|\left[S\left(x_{j}, r_{j}\right)\right]^{1 / k}$

$\leqq \varepsilon^{1 / k} \cdot c\left(1+\varepsilon^{1 / k-1} \cdot 2^{k / k-1} \cdot \alpha(k)^{1 / k-1} \cdot c\right)^{k+1 / k} \alpha(k)^{1 / k}\left\|\tau_{i}\right\|\left[S\left(x_{j}, r_{j}\right)\right]$.

Let $\alpha=2^{k / k-1} \cdot \alpha(k)^{1 / k-1} \cdot c, \beta(\varepsilon)=c\left(1+\varepsilon^{1 / k-1} \cdot 2^{k / k-1} \cdot \alpha(k)^{1 / k-1} \cdot c\right)^{k+1 / k} \cdot \alpha(k)^{1 / k} \cdot$ Notice that $\beta(\varepsilon) \rightarrow c^{k+1 / k+1} \cdot \alpha(k)^{1 / k}$ as $\varepsilon \rightarrow 0$. If we let

$$
\zeta_{i}=\tau_{i}+\sum_{j=1}^{m}\left(\rho_{i, j}-\tau_{i} \cap S_{i, j}\right),
$$

(4) and (6) imply that, for $i \geqq i_{0}$,

$$
W\left(\zeta_{i}-\tau_{i}\right) \leqq \varepsilon^{1 / k} \beta(\varepsilon) M\left(\tau_{i}\right) .
$$

Since

$$
\zeta_{i}=\tau_{i}-\sum_{j=1}^{m} \tau_{i} \cap S_{i, j}+\sum_{j=1}^{m} \rho_{i, j},
$$

it follows from (5) that, for $i \geqq i_{0}$,

$$
M\left(\zeta_{1}\right) \leqq M\left(\tau_{i}-\sum_{j=1}^{m} \tau_{i} \cap S_{i, j}\right)+\varepsilon^{1 / k-1} \alpha M\left(\tau_{i}\right) .
$$

Now, with $U=\mathrm{U}_{j=1}^{m} S\left(x_{j}, r_{j}\right)$, we have from (1)

$$
\begin{aligned}
M\left(\tau_{i}-\sum_{j=0}^{m} \tau_{i} \cap S_{i, j}\right) & =\left\|\tau_{i}\right\|\left(R^{n}\right)-\sum_{j=1}^{m}\left\|\tau_{i}\right\|\left(S_{i, j}\right) \\
& \leqq\left\|\tau_{i}\right\|\left(R^{n}\right)-\sum_{j=1}^{m}\left\|\tau_{i}\right\|\left[S\left(x_{j}, r_{j / 2}\right)\right] \\
& \leqq\left\|\tau_{i}\right\|\left(R^{n}\right)-a \sum_{j=1}^{m}\left\|\tau_{i}\right\|\left[S\left(x_{j}, r_{j}\right)\right] \\
& \leqq\left\|\tau_{i}\right\|\left(R^{n}\right)-a\left\|\tau_{i}\right\|(U) \text { for } i \geqq i_{0} .
\end{aligned}
$$

There exists an integer $i_{1} \geqq i_{0}$ such that for $i \geqq i_{1}$,

$$
||\left|\tau_{i} \|(U)-\mu(U)\right|<\varepsilon ;
$$

therefore, from (8) and (9),

$$
\begin{aligned}
& M\left(\zeta_{i}\right)+a\left\|\tau_{1}\right\|(U) \leqq M\left(\tau_{i}\right)+\varepsilon^{1 / k-1} \cdot \alpha \cdot M\left(\tau_{i}\right), \\
& M\left(\zeta_{i}\right)+a \mu(U) \leqq M\left(\tau_{i}\right)+\varepsilon^{1 / k-1} \cdot \alpha \cdot M\left(\tau_{i}\right)+a \varepsilon .
\end{aligned}
$$

But $\mu(U)>\mu(E)-\varepsilon$, and therefore we finally obtain, for $i \geqq i_{1}$,

$$
M\left(\zeta_{i}\right)+a \mu(E) \leqq M\left(\tau_{i}\right)+\varepsilon^{1 / k-1} \cdot \alpha \cdot M\left(\tau_{i}\right)+2 \alpha \varepsilon .
$$


Hence, from this inequality and (7), it is now clear that we can find a sequence of integral cycles $\psi_{i}$ such that

$$
\begin{gathered}
\lim _{i \rightarrow \infty} W\left(\psi_{i}-\tau\right)=0, \\
\lim _{i \rightarrow \infty} \sup M\left(\psi_{i}\right)+a \mu(E) \leqq \lim _{i \rightarrow \infty} M\left(\tau_{i}\right)=L(\tau),
\end{gathered}
$$

which is a contradiction since $\mu(E)>0$ and $a>0$.

3. The main theorem. In this section, the main theorem, which is concerned with the compactness of integral classes, is established. In the proof, an essential role is played by a decomposition theorem due to Federer [4], which will now be discussed.

Recall from [8; 5.13], that if $A$ is a compact subset of $R^{n}$, $\tau_{i} \in I_{k}(A, 2)$ for $i=1,2, \cdots$, and $\sup \left\{M\left(\tau_{i}\right)+M\left(\partial \tau_{i}\right): i=1,2, \cdots\right\}<\infty$, then there exists a subsequence, $\left\{\tau_{i j}\right\}$, and a $\tau \in W_{k}\left(R^{n}, 2\right)$ such that $W\left(\tau_{i j}-\tau\right) \rightarrow 0$. Of course, it would be desirable to show that $\tau$ is, in fact, a rectifiable class. To this end, assume without loss of generality that $\partial \tau=0$ and let $\left\{\tau_{i}\right\}$ be a sequence of integral cycles for which

$$
\lim _{i \rightarrow \infty} W\left(\tau_{i}-\tau\right)=0 \text { and } \lim _{i \rightarrow \infty} M\left(\tau_{i}\right)=L(\tau) .
$$

Hence, by passing to a suitable subsequence if necessary, we have the existence of a non-negative Radon measure $\mu$ such that $\left\{\left\|\tau_{i}\right\|\right\}$ converges weakly to $\mu$. Then, from [4; §9] we know that $R^{n}$ can be decomposed into four $\mu$-measurable sets $A_{1}, A_{2}, A_{3}, A_{4}$ such that:

(1) $R^{n}=A_{1} \cup A_{2} \cup A_{3} \cup A_{4}$,

(2) $A_{1} \cap A_{2}=A_{1} \cap A_{3}=A_{2} \cap A_{3}=0$,

(3) $A_{1}$ is a countably $k$-rectifiable set and at each point $x \in A_{1}$, there exists a $\mu$-approximate tangent $k$-plane to $A_{1}$ at $x$; for this, (2.8) is needed,

(4) Either $\mu\left(A_{2}\right)=0$ or $A_{2}$ contains no $k$-rectifiable set $B$ for which $\mu(B)>0$,

(5) $L_{k}\left[p\left(A_{2}\right)\right]=0$ for almost all orthogonal projections of $R^{n}$ onto $R^{k}$,

(6) $A_{3}=\left\{x: D_{k}\left(\mu, R^{n}, x\right)=0\right.$ or $\left.D_{k}^{*}\left(\mu, R^{n}, x\right)=\infty\right\}$. Observe that from $2.8, \mu\left[\left\{x: D_{k}\left(\mu, R^{n}, x\right)=0\right\}\right]=0$,

(7) $\mu\left(A_{4}\right)=0$.

Now let $A=A_{1} \cap\left\{x: D_{k}\left(H^{k}, A_{1}, x\right)=1\right\} \cap B$, where $B$ is the set described in the proof of $[8 ; 5.14]$. We now are in a position to state the main theorem.

3.1. THeOREM. Suppose that $H^{k}\left(A_{1}\right)<\infty$ and that $\mu\left(A_{2}\right)=\mu\left(A_{3}\right)=$ 0. Then, there exists a $\mu$-measurable set $E \subset R^{n}$ such that $\mu(A-E)=$ 
0 and

$$
\lim _{i \rightarrow \infty} W\left(\tau_{i}-A \cap E\right)=0 .
$$

Since $A \cap E$ is a Hausdorff $k$-rectifiable set, by [8; $\S 3]$, we can identify $A \cap E$ with a rectifiable class. Hence, the theorem asserts the existence of a rectifiable class to which $\left\{\tau_{i}\right\}$ converges.

For the proof of the theorem, we will need the following lemma.

3.2. LemmA. Suppose that $A$ is a countably k-rectifiable set with $H^{k}(A)<\infty$. Then, for any real number $0<a<1$, and for any real number $b$ such that $b>1$ and $b^{k}<a^{-1}$,

$$
\lim _{r \rightarrow 0} \frac{H^{k}[S(x, r / b) \cap A]}{H^{k}[S(x, r) \cap A]}>a,
$$

for $H^{k}$ almost all $x \in A$.

Proof. Since $D_{k}\left(H^{k}, A, x\right)=1$ for $H^{k}$ almost all $x \in A$, we have the following at all such $x$ :

$$
\lim _{r \rightarrow 0} b^{-k} \frac{H^{k}[S(x, r / b) \cap A]}{(r / b)^{k}} \cdot \frac{r^{k}}{H^{k}[S(x, r) \cap A]}=b^{-k}>a .
$$

Proof of the theorem. It is sufficient to show that the conclusion holds for a subsequence of the given sequence. Passage to subsequences, which often occurs in what follows, will be indicated by words but not notationally. The proof will be divided into four main parts.

Choose $0<\delta<1$ and let $\gamma(B)=H^{k}(A \cap B)$ where $B$ is any $H^{k}$ measurable set. In view of the assumptions and with the aid of [4; (3.8)] we know that $\mu$ is absolutely continuous with respect to \%. Let $P(x)$ be the $\mu$ approximate tangent $k$-plane to $A$ at $x$ and let $K(x, r)$ be the open $n$-cube with center at $x$, side length $2 r$ and one of its $k$-faces parallel to $P(x)$. In this proof, densities will be computed by using these cubes and 2.6 will be used with cubes instead of spheres; this does not change anything. Using the methods of [8; 5.14], for $\varepsilon>0$ we have the existence of a positive number $r_{1}(x, \varepsilon)$ such that for $r \leqq r_{1}(x, \varepsilon)$,

$$
W[P(x) \cap K(x, r))-A \cap K(x, r)]<\varepsilon^{2} / 8 \cdot \beta(k) r^{k}
$$

where $\beta(k)$ is volume of a $k$-cube with side length 2 . Also, if $D(x)=$ $D_{k}^{*}\left(\mu, R^{n}, x\right)$, then for each $x \in A$ there exists a number $r_{0}(x, \varepsilon) \leqq r_{1}(x, \varepsilon)$ such that for $r \leqq r_{0}(x, \varepsilon)$, 


$$
\begin{aligned}
& \gamma[K(x, r)] \geqq(1-\varepsilon) \beta(k) r^{k}, \\
& \mu[K(x, r)]<c(x) \beta(k)(r / b)^{k}, \\
& \mu\left[K(x, r)-S(P(x), \varepsilon / 2 \cdot r]<\varepsilon^{2} 2^{-k} \beta(k) r^{k},\right. \\
& \gamma[K(x, r / b)]>a \gamma[K(x, r)],
\end{aligned}
$$

where $c(x)=b^{k} D(x), a>1-\varepsilon$, and where $b$ is the number provided by 3.2. We will consider only those $r$ for which $u\left[K^{*}(x, r)\right]=$ $\mu\left[K^{*}(x, r / b)\right]=0$, where $K^{*}(x, r)$ denotes the boundary of the cube. Since this omits at most a denumerable number of cubes, we have a Vitali covering of $A$ and therefore, by 2.6, there exists a finite number of disjoint cubes $K\left(x_{1}, r_{1}\right), K\left(x_{2}, r_{2}\right), \cdots, K\left(x_{m}, r_{m}\right)$ such that $\gamma\left[A-\bigcup_{i=1}^{m} K\left(x_{i}, r_{i}\right)\right]<\varepsilon$ and $\mu\left[A-\bigcup_{i=1}^{m} K\left(x_{i}, r_{i}\right)\right]<\varepsilon$. Let $c=$ $\max \left[c\left(x_{1}\right), \cdots, c\left(x_{m}\right)\right], d=c /(b-1)$, and assume $\varepsilon$ to be chosen so as to satisfy the following inequalities, where $c_{1}$ and $c_{2}$ are the constants in $[8 ; 4.6]$ :

$$
\varepsilon<\left[c_{1} 2^{2-2 k} \beta(k)\right]^{-1}, \quad\left[\varepsilon c_{2} 4^{k-1} 2^{1-k} \beta(k)\right]^{k / k-1}+\varepsilon^{2}<\delta^{2} / 8,
$$

(3) $\varepsilon c+\varepsilon L d+3 \varepsilon^{2}<\delta^{2} / 4$ where $L$ is described below,

$$
\varepsilon<1-\delta / 2, \varepsilon c / 1-\varepsilon<\delta, \varepsilon \gamma(A)+\varepsilon-\varepsilon^{2}<\delta .
$$

Part 1. Consider $x_{1}$ and let $x=x_{1}, r=r_{1}$, and $P=P\left(x_{1}\right)$. Since $\mu\left[K^{*}(x, r)\right]=0$ and $\left\|\tau_{i}\right\| \rightarrow \mu$, there exists an integer $i_{0}(r)$ such that for $i \geqq i_{0}(r)$,

$$
\begin{aligned}
& M\left[\tau_{i} \cap K(x, r)\right]<c \beta(k)(r / b)^{k}, \\
& M\left[\tau_{i} \cap(K(x, r)-S(P, \varepsilon / 2 \cdot n))\right]<\varepsilon^{2} 2^{-k} \beta(k) r^{k} .
\end{aligned}
$$

For each $i \geqq i_{0}(r),[8 ; 4.1]$ implies that

$$
\int_{r / b}^{r} M\left[\partial\left(\tau_{i} \cap K(x, s)\right] d s<L c \beta(k)(r / b)^{k},\right.
$$

where $L$ is the Lipschitz constant of the function that defines $K(x, s)$. Therefore, by appealing to Fatou's lemma, there exists a number $t$ between $r / b$ and $r$ and a subsequence $\left\{\tau_{i}\right\}$ (which will still be denoted by $\left.\left\{\tau_{i}\right\}\right)$ such that

$$
\mu\left[K^{*}(x, t)\right]=0
$$

and

$$
\begin{aligned}
M\left[\partial\left(\tau_{i} \cap K(x, t)\right)\right] & \leqq L c \beta(k)^{1} / b-1(r / b)^{k-1} \\
& \leqq L d \beta(k) t^{k-1}, \quad \text { for all } i .
\end{aligned}
$$

Hence, letting $\sigma_{i}=\tau_{i} \cap K(x, t)$ from (1) and (2), we have the following for all elements of a subsequence: 


$$
\begin{aligned}
& M\left(\sigma_{i}\right) \leqq c \beta(k) t^{k}, \\
& M\left(\partial \sigma_{i}\right) \leqq L d \beta(k) t^{k-1}, \\
& M\left[\sigma_{i} \cap(K(x, t)-S(P, \varepsilon / 2 \cdot t))\right] \leqq \varepsilon^{2} 2^{-k} \beta(k) t^{k}, \\
& \gamma[K(x, t)]>a \gamma[K(x, r)], \\
& W[P \cap K(x, t)-A \cap K(x, t)]<\varepsilon^{2} / 8 \cdot \beta(k) t^{k}, \\
& \text { spt } \sigma_{i} \subset \operatorname{closure} K(x, t), \text { spt } \delta \sigma_{i}=K^{*}(x, t) .
\end{aligned}
$$

Let $U_{s}=\{x$ : distance $(x, P)>s\}$. For each $\sigma_{i}$ of the above subsequence, we have from $[8 ; 4.1]$ that

$$
\int_{\varepsilon t / 2}^{\varepsilon t} M\left[\partial\left(\sigma_{i} \cap U_{s}\right)-\left(\partial \sigma_{i}\right) \cap U_{s}\right] d s<\varepsilon^{2} 2^{-k} \beta(k) t^{k},
$$

so that again by appealing to Fatou's lemma, there exists a number $s_{0}$ such that $\varepsilon t / 2<s_{0}<\varepsilon t$ and a subsequence $\left\{\sigma_{i}\right\}$ such that for all members of this subsequence,

$$
M\left[\partial\left(\sigma_{i} \cap U_{s_{0}}\right)-\left(\partial \sigma_{1}\right) \cap U_{s_{0}}\right] \leqq \varepsilon 2^{1-k} \beta(k) t^{k-1} .
$$

Let $K=K(x, t), U=U_{s_{0}}, N=$ closure $\left[K \cap\left(R^{n}-U\right)\right]$ and note that

$$
\text { spt } p_{\sharp}\left[\left(\partial \sigma_{i}\right) \cup N\right] \subset P \cap K^{*}
$$

where $p: R^{n} \rightarrow P$ is the orthogonal projection. If we let (7) $\theta_{i}=\partial\left(\sigma_{i} \cap N\right)-\left(\partial \sigma_{i}\right) \cap N=\partial\left(\sigma_{i} \cap U\right)-\left(\partial \sigma_{i}\right) \cap U$ and $\chi_{i}=p_{\sharp}\left(\sigma_{i} \cap N\right)$, then in the notation of $[8 ; 4.6]$ with $A=$ closure $(P \cap K)$ and $B=$ $P \cap K^{*}$, we have from (3), (5), and (6),

$$
\begin{aligned}
c_{1} M\left(\partial \chi_{i} \cap K\right) & =c_{1} M\left[p_{\ddagger}\left(\theta_{i}\right) \cap K\right] \leqq c_{1} M\left[p_{\sharp}\left(\theta_{i}\right)\right] \\
& \leqq c_{1} 2^{1-k} \varepsilon \beta(k) t^{k-1} \leqq(t / 2)^{k-1}
\end{aligned}
$$

Hence, by [8; 4.6], there exists $\lambda_{i} \in I_{k}(A, 2)$ such that

$$
\begin{aligned}
& \operatorname{spt}\left(\partial \chi_{i}-\partial \lambda_{i}\right) \subset B, \\
& M\left(\lambda_{i}\right)^{k-1 / k} \leqq \varepsilon c_{2} 4^{k-1} 2^{1-k} \beta(k) t^{k-1} .
\end{aligned}
$$

If we let $\psi_{i}=\chi_{i}-\lambda_{i}$, then we have

$$
\begin{aligned}
& \text { spt } \psi_{i} \subset \text { closure } P \cap K, \\
& \text { spt } \partial \psi_{i} \subset P \cap K^{*}, \\
& M\left(\psi_{i}-\chi_{i}\right) \leqq \varepsilon^{k / k-1}\left[c_{2} 4^{k-1} 2^{1-k} \beta(k)\right]^{k / k-1} t^{k} .
\end{aligned}
$$

Hence, we will consider the two following possibilities: $\psi_{i}=0$ for all but finitely many $i$ or $\psi_{i}=P \cap K$ for all $i$ of a subsequence.

Case 1. Suppose $\psi_{i}=P \cap K$ for some subsequence. Then from (4), (8), and (3), 


$$
\begin{aligned}
M\left[P \cap K-p_{\sharp}\left(\sigma_{i}\right)\right] & \leqq M\left[P \cap K-p_{\sharp}\left(\sigma_{i} \cap N\right)\right]+M\left[p_{\sharp}\left(\sigma_{i} \cap U\right)\right] \\
& \leqq M\left(\psi_{i}-\chi_{i}\right)+M\left[p_{\sharp}\left(\sigma_{i} \cap U\right)\right] \\
& \leqq \varepsilon^{k / k-1}\left[c_{2} 4^{k-1} 2^{1-k} \beta(k)\right]^{k / k-1} t^{k}+\varepsilon^{2} / 2^{k} \cdot \beta(k) t^{k} \\
& \leqq \delta^{2} / 8 \cdot \beta(k) t^{k}
\end{aligned}
$$

Therefore, (4) implies $W\left[p_{\sharp}\left(\sigma_{i}\right)-A \cap K\right]<\delta^{2} / 4 \cdot \beta(k) t^{k}$. Also from (4), (3)

$$
\begin{aligned}
W\left[p_{\sharp}\left(\sigma_{i}\right)-\sigma_{i}\right] & \leqq W\left[p\left(\sigma_{i} \cap N\right)-\sigma_{i} \cap N\right]+W\left[p_{\sharp}\left(\sigma_{i} \cap U\right)-\sigma_{i} \cap U\right] \\
& \leqq \varepsilon t N\left(\sigma_{i} \cap N\right)+2 M\left(\sigma_{i} \cap U\right) \\
& \leqq \varepsilon t\left[c \beta(k) t^{k}+L d \beta(k) t^{k-1}\right]+\varepsilon^{2} 2^{1-k} \beta(k) t^{k} \\
& \leqq \delta^{2} / 4 \cdot \beta(k) t^{k} .
\end{aligned}
$$

Therefore, from (2), (3), and (4),

$$
W\left[\tau_{i} \cap K-A \cap K\right]<\delta^{2} / 2 \cdot \beta(k) t^{k} \leqq \delta K(x, t)
$$

for all members of a suitable subsequence. Now repeat the entire above procedure to the cube $K\left(x_{2}, r_{2}\right)$, but using the subsequence that was finally obtained at the end of case 1 .

Case 2. In the event that $\psi_{i}=0$ for all but finitely many $i$, repeat the entire above procedure to the cube $K\left(x_{2}, r_{2}\right)$ but using the subsequence that corresponds to $\psi_{i}=0$.

Part 2. By repeating the procedure in part $1 m$ times, we obtain cubes $K\left(x_{1}, t_{1}\right), \cdots, K\left(x_{j}, t_{j}\right), K\left(x_{j+1}, t_{j+1}\right), \cdots, K\left(x_{m}, t_{m}\right)$ and a subsequence, $\left\{\tau_{i}\right\}$ such that, for all members of this subsequence,

$$
\sum_{l=1}^{j} W\left[\tau_{i} \cap K\left(x_{l}, t_{l}\right)-A \cap K\left(x_{l}, t_{l}\right)\right]<\delta^{2} / 2 \cdot \beta(k) \sum_{l=1}^{j} t_{l}^{k} \leqq \delta \gamma(A),
$$

and such that case 2 of step 1 applies to the cubes $K\left(x_{j+l}, t_{j+l}\right), \cdots$, $K\left(x_{m}, t_{m}\right)$. Now, using the same notation as above except for the addition of superscripts to denote that cube which is under consideration, we have that $\psi_{i}^{q}=0$ for all $i$ and for $q=j+1, j+2, \cdots, m$. This implies that $M\left[p_{\sharp}^{q}\left(\sigma_{i}^{q} \cap N^{q}\right)\right]<\delta^{2} / 8 \cdot \beta(k) t_{q}^{k}$, where $p^{q}: R^{n} \rightarrow P\left(x_{q}\right)$ is the orthogonal projection. Define $A_{\delta}=\bigcup_{l=1}^{j} K\left(x_{l}, t_{l}\right)$ and $B_{\delta}=$ $\bigcap_{l=j+1}^{m} K\left(x_{l}, t_{l}\right)$ and let $\omega_{i}^{q}=h_{\sharp}^{q}\left[I \times \partial\left(\sigma_{i}^{q} \cap N^{q}\right)\right], \quad \zeta_{i}^{q}=h_{\sharp}^{q}\left[I \times\left(\sigma_{i}^{q} \cap N^{q}\right)\right]$ where $h_{\ddagger}^{q}$ is the linear homotopy from the identity to the projection map $p^{q}$. If we let

$$
\tau_{i}^{\delta}=\sum_{q=j+l}^{m} \tau_{i} \cap\left[R^{n}-K\left(x_{q}, t_{q}\right)\right]+\sum_{q=j+l}^{m}\left[\omega_{i}^{q}+p_{q}^{q}\left(\sigma_{i}^{q} \cap N^{q}\right)+\sigma_{i}^{q} \cap U^{q}\right],
$$

then $\partial \tau_{i}^{\delta}=0$ and from (7), (5), (8), (4), (3), and (2), 


$$
\begin{aligned}
M\left(\tau_{i}^{\delta}\right) \leqq & M\left(\tau_{i}\right)-M\left(\tau_{i} \cap B_{\delta}\right)+\varepsilon t\left[\sum_{q=j+1}^{m} 2 \varepsilon \beta(k) t_{q}^{k-1}+L d \beta(k) t_{q}^{k-1}\right] \\
& +\delta^{2} / 4 \sum_{q=j+1}^{m} \beta(k) t_{q}^{k}+\varepsilon^{2} \sum_{q=j+1}^{m} \beta(k) t_{q}^{k} \\
\leqq & M\left(\tau_{i}\right)-M\left(\tau_{i} \cap B_{\delta}\right)+\delta^{2} / 2 \sum_{q=j+1}^{m} \beta(k) t_{q}^{k} \\
\leqq & M\left(\tau_{i}\right)-M\left(\tau_{i} \cap B_{\delta}\right)+\delta^{2} / 2(1-\varepsilon) \cdot \gamma\left(\beta_{\delta}\right) \\
\leqq & M\left(\tau_{i}\right)-M\left(\tau_{i} \cap B_{\delta}\right)+\delta \gamma(A) .
\end{aligned}
$$

Since $\left\|\tau_{i}\right\| \rightarrow \mu$, there exists a subsequence of the one above such that, for all members of this subsequence,

$$
M\left(\tau_{i}^{\delta}\right) \leqq M\left(\tau_{i}\right)-\mu\left(B_{\delta}\right)+2 \delta \gamma(A) .
$$

Also,

$$
\tau_{i}-\tau_{i}^{\delta}=\sum_{q=j+1}^{m} \partial \zeta_{i}^{q}
$$

and therefore, from (3),

$$
\begin{aligned}
W\left(\tau_{i}-\tau_{i}^{\delta}\right) & \leqq \sum_{q=j+1}^{m} M\left(\zeta_{i}^{q}\right) \leqq \varepsilon \sum_{q=j+1}^{m} c \beta(k) t_{q}^{k} \\
& \leqq \varepsilon c / 1-\varepsilon \cdot \gamma\left(B_{\delta}\right)<\delta \gamma(A) .
\end{aligned}
$$

Observe, with the help of (4), that

$$
\begin{aligned}
\gamma\left(A_{\delta} \cup B_{\delta}\right) & \geqq a \gamma\left[\bigcup_{i=1}^{m} K\left(x_{i}, r_{i}\right)\right] \geqq a[\gamma(A)-\varepsilon] \\
& \geqq(1-\varepsilon)[\gamma(A)-\varepsilon] \geqq \gamma(A)-\delta .
\end{aligned}
$$

Thus, in summary of what has been done so far, we have, for every $\delta>0$, the existence of sets $A_{\delta}$ and $B_{\delta}$ which are the finite union of disjoint open cubes and the existence of a subsequence $\left\{\tau_{i}\right\}$ and a sequence $\left\{\tau_{i}^{s}\right\}$ such that

$$
\begin{aligned}
& \gamma\left[A-\left(A_{\delta} \cup B_{\delta}\right)\right]<\delta, \\
& W\left(\tau_{i} \cap A_{\delta}-A \cap A_{\delta}\right)<\delta \gamma(A), \\
& W\left(\tau_{i}-\tau_{i}^{\delta}\right)<\delta \gamma(A), \\
& M\left(\tau_{i}^{\delta}\right) \leqq M\left(\tau_{i}\right)-\mu\left(B_{\delta}\right)+2 \delta \gamma(A) \quad \text { for all } i .
\end{aligned}
$$

Now by letting $\delta \rightarrow 0$ and by using Cantor's diagonal process, we can infer that $\lim _{\delta \rightarrow 0} \sup \mu\left(B_{\delta}\right)=0$ since $M\left(\tau_{i}\right) \rightarrow L(\tau)$. This implies, along with the fact that $\mu$ is absolutely continuous with respect to $\gamma$, that for every $\delta>0$, there exists a set $A_{\delta}$, which is the union of a finite number of disjoint open cubes, and a subsequence $\left\{\tau_{i}\right\}$ such that $\mu\left(R^{n}-A_{\delta}\right)<\delta$ and $W\left(\tau_{i} \cap A_{\delta}-A \cap A_{\delta}\right)<\delta$ for all $i$. 
Part 3. Choose $0<\delta<1$ and let $\left\{\delta_{i}\right\}$ be a sequence of real numbers tending to zero with $\sum_{i=1}^{\infty} \delta_{i}<\delta / 3$. Part 2 supplies a set $A_{\delta_{1}}$. Now repeat the procedures in parts 1 and 2 to the set $A-A_{\delta_{1}}$ with the restriction that only those cubes that do not intersect the closure of $A_{\delta_{1}}$ should be considered. Since the $\mu$ measure of the frontier of $A_{\delta_{1}}$ is zero, those cubes with centers on the frontier of $A_{\delta_{1}}$ need not be considered. Also, the subsequence that is obtained for the set $A_{\delta_{1}}$ is the one that should be used in the procedure for $A-A_{\delta_{1}}$. Hence, we will obtain a subsequence of the sequence obtained for $A_{\delta_{1}}$ and a set $A_{\delta_{2}}$ such that $A_{\delta_{2}}$ is the finite union of open disjoint cubes with $A_{\delta_{2}} \subset R^{n}-A_{\delta_{1}}, \quad \mu\left[R^{n}-\left(A_{\delta_{1}} \cup A_{\delta_{2}}\right)\right]<\delta_{2}$, and $W\left(\tau_{i} \cap A_{\delta_{2}}-A \cap A_{\delta_{2}}\right)<\delta_{2}$ for all $i$. Continue this process and let $H_{\delta}=\bigcup_{i=1}^{\infty} A_{\delta_{i}}$. Then, $\mu\left(A-H_{\delta}\right)=0$ and by employing Cantor's diagonal process, we obtain the following: if $S_{j}=\bigcap_{i=1}^{j} A_{\delta i}$, then there exists an integer $j_{0}$ such that for $j \geqq j_{0}$,

$$
\gamma\left(A \cap H_{\delta}-A \cap S_{j}\right)<\delta / 3
$$

and

$$
\mu\left(R^{n}-S_{j}\right)=\mu\left(A \cap H_{\delta}-A \cap S_{j}\right)<\delta / 3 .
$$

Hence, $M\left(A \cap H_{\delta}-A \cap S_{j_{0}}\right)<\delta / 3$. Since $\left\|\tau_{i}\right\| \rightarrow \mu$ and since $S_{j_{0}}$ is open, there exists one integer $i_{0}\left(j_{0}\right)$ such that for $i \geqq i_{0}\left(j_{0}\right)$, $M\left(\tau_{i}-\tau_{i} \cap S_{j_{0}}\right)<\delta / 3$. Therefore, for $i \geqq i_{0}\left(j_{0}\right)$,

$$
\begin{aligned}
W\left(\tau_{i}-A \cap H_{\delta}\right) \leqq & W\left(\tau_{i}-\tau_{i} \cap S_{j_{0}}\right)+W\left(\tau_{i} \cap S_{j_{0}}-A \cap S_{j_{0}}\right) \\
& +W\left(A \cap S_{j_{0}}-A \cap H_{\delta}\right) \\
\leqq & \delta / 3+\sum_{i=1}^{j_{0}} \delta_{i}+\delta / 3<\delta .
\end{aligned}
$$

We now have, for every $\delta>0$, an open set $H_{\delta}$ and a subsequence $\left\{\tau_{i}\right\}$ such that $\mu\left(A-H_{\delta}\right)=0$ and $W\left(\tau_{i}-A \cap H_{\delta}\right)<\delta$ for all $i$.

Part 4. Choose $\delta>0$ and again let $\left\{\delta_{1}\right\}$ be a sequence of real numbers tending to zero. After obtaining the set $H_{\delta_{1}}$, repeat parts 1,2 , and 3 to the set $A \cap H_{\delta_{1}}$ and to the sequence that was obtained for $A \cap H_{\delta_{1}}$. Since $H_{\delta_{1}}$ is open, we can require that $H_{\delta_{2}} \subset H_{\delta_{1}}$. Continue this process and let $E=\bigcap_{i=1}^{\infty} H_{\delta_{1}}$ to obtain $\mu(A-E)=0$. By employing Cantor's diagonal process, we obtain a sequence $\left\{\tau_{i}\right\}$ such that $W\left(\tau_{i}-A \cap H_{\delta_{j}}\right)<\delta_{j}$ for large $i$. Choose $j_{0}$ such that $\delta_{j_{0}}<\delta / 2$ and $\gamma\left(A \cap H_{\delta_{j_{0}}}-A \cap E\right)<\delta / 2$. Thus

$$
\begin{aligned}
W\left(\tau_{i}-A \cap E\right) & \leqq W\left(\tau_{i}-A \cap H_{\delta_{\delta_{0}}}\right)+W\left(A \cap H_{\delta_{\delta_{0}}}-A \cap E\right) \\
& \leqq \delta / 2+\gamma\left(A \cap H_{\delta_{\delta_{0}}}-A \cap E\right)<\delta \text { for large } i,
\end{aligned}
$$

and therefore the conclusion of the theorem follows. 


\section{BIBLIOGRAPHY}

1. A. S. Besicovitch, $A$ general form of the covering principle and relative differentiation of additive functions, I, II, Proc. Cambridge Philos. Soc., 41 (1945), 103-110; 42 (1946), 1-10.

2. N. Bourbaki, Integration, Actualites Sci. Ind. No. 1175, 1244, Hermann, Paris, 1952, 1956.

3. H. Federer and W. H. Fleming, Normal and integral currents, Ann. of Math., (2) 72 (1960), 458-520.

4. H. Federer, The $(\varphi, k)$ rectifiable subsets of $n$ space, Trans. Amer. Math. Soc., 62 (1947), 114-192.

5. A. P. Morse, Perfect blankets, Trans. Am. Math, Soc., 61 (1947), 418-442.

6. E. R. Reifenberg, Solution of the Plateau problem for m-dimensional surfaces of varying topological type, Acta Math., 104 (1960) 1-91.

7. H. Whitney, Geometric integration theory, Princeton University Press, Princeton, N. J., 1957.

8. W. P. Ziemer, Integral currents $\bmod 2$, Trans, Amer. Math. Soc., 105 (1962), 496524.

INDIANA UNIVERSITY 


\section{PACIFIC JOURNAL OF MATHEMATICS}

\section{EDITORS}

RalPh S. Phillips

Stanford University

Stanford, California

M. G. Arsove

University of Washington

Seattle 5 , Washington
J. Dugundu

University of Southern California

Los Angeles 7, California

Lowell J. Paige

University of California

Los Angeles 24, California

\section{ASSOCIATE EDITORS}
E. F. BECKENBACH
D. DERRY
H. L. ROYDEN
E. G. STRAUS
T. M. CHERRY
M. OHTSUKA
E. SPANIER
F. WOLF

\section{SUPPORTING INSTITUTIONS}

\author{
UNIVERSITY OF BRITISH COLUMBIA \\ CALIFORNIA INSTITUTE OF TECHNOLOGY \\ UNIVERSITY OF CALIFORNIA \\ MONTANA STATE UNIVERSITY \\ UNIVERSITY OF NEVADA \\ NEW MEXICO STATE UNIVERSITY \\ OREGON STATE UNIVERSITY \\ UNIVERSITY OF OREGON \\ OSAKA UNIVERSITY \\ UNIVERSITY OF SOUTHERN CALIFORNIA
}

\author{
STANFORD UNIVERSITY \\ UNIVERSITY OF TOKYO \\ UNIVERSITY OF UTAH \\ WASHINGTON STATE UNIVERSITY \\ UNIVERSITY OF WASHINGTON \\ AMERICAN MATHEMATICAL SOCIETY \\ CALIFORNIA RESEARCH CORPORATION \\ SPACE TECHNOLOGY LABORATORIES \\ NAVAL ORDNANCE TEST STATION
}

Mathematical papers intended for publication in the Pacific Journal of Mathematrcs should be typewritten (double spaced), and the author should keep a complete copy. Manuscripts may be sent to any one of the four editors. All other communications to the editors should be addressed to the managing editor, L. J. Paige at the University of California, Los Angeles 24, California.

50 reprints per author of each article are furnished free of charge; additional copses may be obtained at cost in multiples of 50 .

The Pacific Journal of Mathematics is published quarterly, in March, June, September, and December. Effective with Volume 13 the price per volume (4 numbers) is $\$ 18.00$; single issues, $\$ 5.00$. Special price for current issues to individual faculty members of supporting institutions and to individual members of the American Mathematical Society: $\$ 8.00$ per volume; single issues $\$ 2.50$. Back numbers are available.

Subscriptions, orders for back numbers, and changes of address should be sent to Pacific Journal of Mathematics, 103 Highland Boulevard, Berkeley 8, California.

Printed at Kokusai Bunken Insatsusha (International Academic Printing Co., Ltd.), No. 6 , 2-chome, Fujimi-cho, Chiyoda-ku, Tokyo, Japan.

\section{PUBLISHED BY PACIFIC JOURNAL OF MATHEMATICS, A NON-PROFIT CORPORATION}

The Supporting Institutions listed above contribute to the cost of publication of this Journal, but they are not owners or publishers and have no responsibility for its content or policies. 


\section{Pacific Journal of Mathematics}

\section{Vol. 13, No. $4 \quad$ June, 1963}

Dallas O. Banks, Bounds for eigenvalues and generalized convexity ........... 1031

Jerrold William Bebernes, A subfunction approach to a boundary value problem for

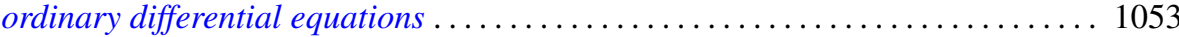

Woodrow Wilson Bledsoe and A. P. Morse, A topological measure construction . . . 1067

George Clements, Entropies of several sets of real valued functions . . . . . . . . . 1085

Sandra Barkdull Cleveland, Homomorphisms of non-commutative *-algebras . . . . . 1097

William John Andrew Culmer and William Ashton Harris, Convergent solutions of

ordinary linear homogeneous difference equations . . . . . . . . . . . . . . . 1111

Ralph DeMarr, Common fixed points for commuting contraction mappings . . . . . . 1139

James Robert Dorroh, Integral equations in normed abelian groups . . . . . . . . 1143

Adriano Mario Garsia, Entropy and singularity of infinite convolutions . . . . . . . 1159

J. J. Gergen, Francis G. Dressel and Wilbur Hallan Purcell, Jr., Convergence of extended Bernstein polynomials in the complex plane ................. 1171

Irving Leonard Glicksberg, A remark on analyticity of function algebras . . . . . . 1181

Charles John August Halberg, Jr., Semigroups of matrices defining linked operators

with different spectra ................................. 1187

Philip Hartman and Nelson Onuchic, On the asymptotic integration of ordinary

differential equations . . . . . . . . . . . . . . . . . . . . . . . . . . . . 1193

Isidore Heller, On a class of equivalent systems of linear inequalities . . . . . . . . . 1209

Joseph Hersch, The method of interior parallels applied to polygonal or multiply

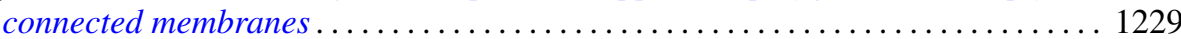

Hans F. Weinberger, An effectless cutting of a vibrating membrane . . . . . . . . . . 1239

Melvin F. Janowitz, Quantifiers and orthomodular lattices ....

Samuel Karlin and Albert Boris J. Novikoff, Generalized convex inequalities . .

Tilla Weinstein, Another conformal structure on immersed surfaces of negative

curvature.

Gregers Louis Krabbe, Spectral permanence of scalar operators

Shige Toshi Kuroda, Finite-dimensional perturbation and a representaion of

scattering operator.

Marvin David Marcus and Afton Herbert Cayford, Equality in certain

inequalities

Joseph Martin, A note on uncountably many disks .

Eugene Kay McLachlan, Extremal elements of the convex cone of semi-norms . . . . 1335

John W. Moon, An extension of Landau's theorem on tournaments . .

Louis Joel Mordell, On the integer solutions of $y(y+1)=x(x$

Kenneth Roy Mount, Some remarks on Fitting's invariants .....

Miroslav Novotný, Über Abbildungen von Mengen ............

Robert Dean Ryan, Conjugate functions in Orlicz spaces.

John Vincent Ryff, On the representation of doubly stochastic operators . . . . . . . . 1379

Donald Ray Sherbert, Banach algebras of Lipschitz functions .

James McLean Sloss, Reflection of biharmonic functions across analytic boundary

conditions with examples.

L. Bruce Treybig, Concerning homogeneity in totally ordered, connected topological space....

John Wermer, The space of real parts of a function algebra...

James Juei-Chin Yeh, Orthogonal developments of functionals and related theorems

in the Wiener space of functions of two variables......... 